\title{
High dose ropivacaine-induced toxicity after infraclavicular block
}

\author{
Chun Woo Yang ${ }^{1}$, Po Soon Kang ${ }^{2}$, Hee Uk Kwon², and Dae Jin Lim³ \\ Department of Anesthesiology and Pain Medicine, ${ }^{1}$ Hongseong Medical Center, Hongseong, ${ }^{2}$ Konyang University Hospital, Daejeon, \\ ${ }^{3}$ Inha University Hospital, Incheon, Korea
}

Local anesthetic toxicity is an uncommon but welldocumented complication of regional anesthesia. The mechanism of local anesthetic toxicity from a peripheral nerve block can be due to either a direct intravascular injection or from systemic absorption of a toxic dose of local anesthetics.

We report two cases of local anesthetic toxicity, including central nervous system toxicity without any cardiac toxicity, following accidental overdose of ropivacaine for infraclavicular block.

The first patient was a 36-year-old female (ASA I, $52 \mathrm{~kg}$, and $160 \mathrm{~cm}$ ), who presented for surgery of the right forearm under infraclavicular block. There was no medical history of neurological or cardiac disease. Physical examination and electrocardiogram (ECG) were unremarkable.

The patient did not receive any sedatives before surgery. In the operating room, standard monitoring (pulse oximetry, noninvasive blood pressure cuff, and ECG) was applied. Using an insulated needle (Stimuplex ${ }^{\circledR}$ A, B. Braun, Germany) and a nerve stimulator (Stimuplex ${ }^{\circledR}$ HNS 12, B. Braun, Germany), a vertical infraclavicular block was performed to provide anesthesia [1]. Once an ulnar nerve response with a current of $0.5 \mathrm{~mA}$ was obtained, a total dose of $40 \mathrm{ml}$ of $0.75 \%$ ropivacaine (300 mg; $5.77 \mathrm{mg} / \mathrm{kg}$ ) without epinephrine was slowly injected with negative aspiration in $5 \mathrm{ml}$ increments. Verbal communication was made during injection, and no early signs of systemic toxicity were noted. Ten minutes after the injection, sensory block was complete in the right arm. About twenty minutes after the injection, complete motor block was achieved in the radial, median, ulnar, and musculocutaneous nerves.

Twenty-four minutes after the injection, the patient complained of numbness in the tongue, nausea, and dizziness. During this time, her blood pressure, heart rate, and oxygen saturation was 156/102 $\mathrm{mmHg}, 81$ beats/min, and 98\%, respectively. No changes were observed on the ECG. Suspecting ropivacaine-induced toxicity, $50 \mathrm{mg}$ of thiopental sodium was administered for seizure prophylaxis, and supplemental oxygen was given via face mask. She recovered over 10 minutes. The patient had no awareness of the incident and was informed. The decision was made to perform surgery and further sedation was given with continuous infusion of propofol. The patient made an uneventful postoperative recovery.

The second patient was a 60-year-old female (ASA I, $47 \mathrm{~kg}$, and $142 \mathrm{~cm}$ ) who was scheduled for surgery of the right forearm under infraclavicular block. She was in good general health, with no significant past medical history and in particular, no history of convulsions or epilepsy. She was taking no medications and had no allergies.

No sedation was used for the block placement. A vertical infraclavicular block was performed with the same technique as described in Case 1. At the first attempt, blood was obtained, and the needle was withdrawn. After shifting of the puncture site in a $1 \mathrm{~cm}$ lateral direction, an ulnar nerve response of fingers with a current of $0.5 \mathrm{~mA}$ was obtained and a total dose of $40 \mathrm{ml}$ of $0.75 \%$ ropivacaine (300 $\mathrm{mg} ; 6.38 \mathrm{mg} / \mathrm{kg}$ ) without epinephrine was slowly injected in $5 \mathrm{ml}$ increments with gentle aspirations between doses. No spontaneous blood return was

Corresponding author: Po Soon Kang, M.D., Department of Anesthesiology and Pain Medicine, School of Medicine, Konyang University, 685, Gasuwon-dong, Seo-gu, Daejeon 302-718, Korea. Tel: 82-42-600-9316, Fax: 82-42-545-2132, E-mail: kangpo1026@naver.com

(c) This is an open-access article distributed under the terms of the Creative Commons Attribution Non-Commercial License (http:// creativecommons.org/licenses/by-nc/3.0/), which permits unrestricted non-commercial use, distribution, and reproduction in any medium, provided the original work is properly cited. 
seen, and no blood could be aspirated. Verbal contact was maintained during the performance of the block. Successful motor and sensory block of the upper limb occurred within 5 min of the injection.

Twenty-eight minutes after the injection, the patient became disoriented and drowsy. Immediately thereafter, she developed tonic-clonic seizure activity. Supplemental oxygen was immediately given via face mask. Propofol $40 \mathrm{mg}$ was administered, resulting in termination of the seizure. During this time, her blood pressure, heart rate, and oxygen saturation was 151/88 $\mathrm{mmHg}$, 66 beats/min, and 100\%, respectively. No arrhythmias were seen on the ECG. Fifteen minutes later, the patient regained consciousness, had no recall of the preceding events, had no sequelae, and was informed. As the patient remained clinically stable and her mental status had returned to baseline, a decision was made to proceed with surgery, which was uneventful.

A peripheral nerve block, such as brachial plexus block, generally requires the use of relatively high doses of local anesthetics. Ropivacaine is an amide local anesthetic with a long duration of action providing increased safety as compared to racemic bupivacaine, because of the reduced potential for cardiovascular and central nervous system toxicity. The lower toxicity of ropivacaine may be advantageous for brachial plexus blocks, enabling large doses to be used. Subsequently, brachial plexus block induced by doses up to $40 \mathrm{ml}$ of $0.75 \%$ ropivacaine (300 mg) provided excellent anesthesia without local anesthetic toxicity [2].

The maximum recommended dose of ropivacaine for brachial plexus block in Finland and the United States is 300 mg [3]. However, standard doses can not be applied to every patient.

In our cases, $300 \mathrm{mg}$ (Case 1, $5.77 \mathrm{mg} / \mathrm{kg}$; Case 2, $6.38 \mathrm{mg} /$ $\mathrm{kg}$ ) of ropivacaine was used for infraclavicualr block, and local anesthetic toxicities occurred after 24 and $28 \mathrm{~min}$, respectively. Plasma ropivacaine levels were, unfortunately, not measured in the cases presented here. However, the delayed onset of the symptoms and complete brachial plexus block suggest a nonintravascular injection, indicating rapid absorption of a large dose of ropivacaine. Therefore, $300 \mathrm{mg}$ of ropivacaine may have been excessive in these patients, although the same amount was used uneventfully in a previous study [2]. Several previous studies have reported $[4,5]$ that $300 \mathrm{mg}$ of ropivacaine could produce local anesthetic toxicity limited to the central nervous system. A review by Rosenberg et al. [3] suggested that because current recommendations regarding maximum doses of local anesthetics are not evidence based, the dose of local anesthetics should be block-specific, site-specific, and patientspecific.

In summary, we have presented 2 cases of local anesthetic toxicity induced by high doses (300 $\mathrm{mg}$ ) of ropivacaine. It is difficult to recommend a safe maximum dose of ropivacaine, but dose calculations must be made on an individual basis, taking into account the sex, weight, and physical status of the patient.

\section{References}

1. Yang CW, Kwon HU, Cho CK, Jung SM, Kang PS, Park ES, et al. A comparison of infraclavicular and supraclavicular approaches to the brachial plexus using neurostimulation. Korean J Anesthesiol 2010; 58: 260-6.

2. Raeder JC, Drøsdahl S, Klaastad O, Kvalsvik O, Isaksen B, Strømskag $\mathrm{KE}$, et al. Axillary brachial plexus block with ropivacaine $7.5 \mathrm{mg} / \mathrm{ml}$. A comparative study with bupivacaine $5 \mathrm{mg} / \mathrm{ml}$. Acta Anaesthesiol Scand 1999; 43: 794-8.

3. Rosenberg PH, Veering BT, Urmey WF. Maximum recommended doses of local anesthetics: a multifactorial concept. Reg Anesth Pain Med 2004; 29: 564-75.

4. Kimura Y, Kamada Y, Kimura A, Orimo K. Ropivacaine-induced toxicity with overdose suspected after axillary brachial plexus block. J Anesth 2007; 21: 413-6.

5. Ala-Kokko TI, Löppönen A, Alahuhta S. Two instances of central nervous system toxicity in the same patient following repeated ropivacaine-induced brachial plexus block. Acta Anaesthesiol Scand 2000; 44: 623-6. 